\section{CMOS exponential function generator}

Weihsing Liu and Shen-Iuan Liu

A new CMOS exponential function generator is presented. The proposed circuit is compact, with low power and wide dynamic range. The proposed circuit has been fabricated in a $0.50 \mu \mathrm{m}$ CMOS process. Experimental results show that the output range of the proposed exponential function generator can be mote than $15 \mathrm{~dB}$ with the liner error less than $\pm 0.5 \mathrm{~dB}$. The supply voltage is $\pm 1.5 \mathrm{~V}$ and the power dissipation is less than $0.4 \mathrm{~mW}$. Experimental results are given to demonstrate the proposed circuit.

Introduction: The exponential function generator is one of the important building blocks in a variable gain amplifier (VGA) [1]. However, unlike the BJT device, there is no intrinsic logarithmic device working in saturation for CMOS technologies. To realise the exponential function, the approximated Taylor's series expansion [2-4] can be utilised. Alternatively, a pseudo-exponential function $[5,6]$ can be used to generate the required exponential function. In this Letter, a new CMOS pseudo-exponential function generator is presented and the experimental results are given to verify the theoretical analysis.

Design principle and circuit implementation: The pseudo-exponential function $f(x)$, which can mimic the exponential function $\exp (2 x)$ $[6]$, is expressed as

$$
f(x)=\frac{1+x}{1-x} \simeq \exp (2 x) \text { if }|x|<1
$$

The comparison between the pseudo-exponential function and $\exp (2 x)$ is shown in Fig. 1. The Figure shows that as $x$ varies from -0.4 to 0.4 , the output dynamic range can be more than $15 \mathrm{~dB}$ while the difference between $f(x)$ and $\exp (2 x)$ is within $\pm 0.5 \mathrm{~dB}$.

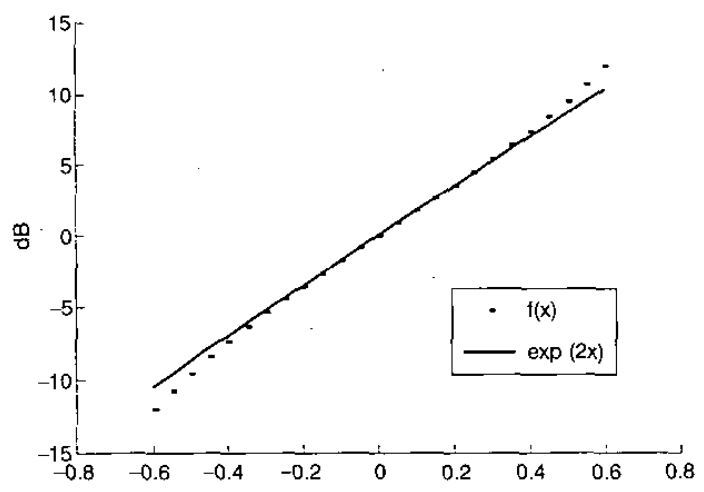

Fig. 1 Comparison between function $f(x)=(1+x) /(l-x)$ and $\exp (2 x)$

The proposed CMOS pseudo-exponential function generator is shown Fig. 2. Assume that both transistors $\mathrm{Ml}$ and $\mathrm{M} 2$ are biased in the triode region without body effect. The source currents $I_{1}$ and $I_{2}$ can be expressed as

$$
I_{1}=\frac{K_{n 1}}{2}\left(2\left(-V_{S S}-V_{I n 1}\right) V_{D S 1}-V_{D S 1}^{2}\right)
$$

and

$$
I_{2}=I_{b}+I_{\text {in }}=\frac{K_{n 2}}{2}\left(2\left(V_{G 2}-V_{S S}-V_{T n 2}\right) V_{D S 2}-V_{D S 2}^{2}\right)
$$

where $I_{b}$ is a reference current, $K_{n, 2}$ are the transconductance parameters and $V_{T n 1.2}$ are the threshold voltages of $\mathrm{Ml}$ and $\mathrm{M} 2$, respectively. The current mirror, M5 and M6, is used to duplicate the current $I_{1}$ as

$$
I_{1}=I_{3}=I_{4}
$$

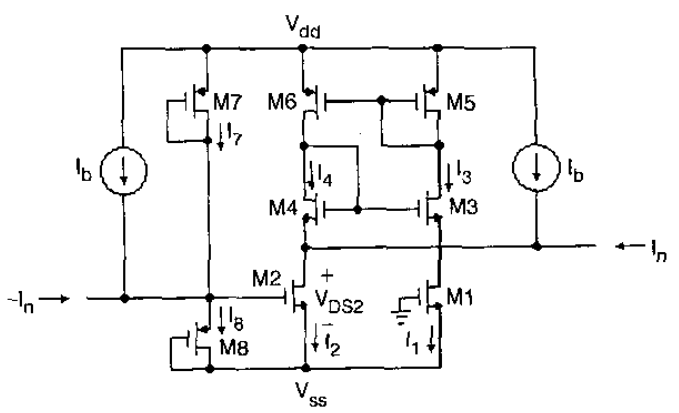

Fig. 2 Proposed exponential function generator

Assume that M3 and M4 are perfectly matched (i.e. $K_{n 3}=K_{n 4}$ and $\left.V_{T n 3}=V_{T n 4}\right)$ and both are biased in saturation. According to the squarelaw characteristics of MOSFETs, the following equation can be obtained

$$
V_{G S 3}=V_{G S 4}=\sqrt{\frac{2 I_{1}}{K_{n 3}}}+V_{T n 3}
$$

According to (5) and $V_{G S 3}+V_{D S 1}=V_{G S 4}+V_{D S 2}$, one can have

$$
\dot{V}_{D S 1}=V_{D S 2}
$$

Since the source voltages of M3 and M4 are equal (i.e. $V_{S B 3}=V_{S_{B 4}}$ ), one can find $V_{T n 3}=V_{T n 4}$. Substituting (2), (4) and (6) into (3) and assuming that $\mathrm{M} 1$ and $\mathrm{M} 2$ are perfectly matched (i.e. $K_{n 1}=K_{n 2}=K_{n}$ and $V_{T n 1}=V_{T n 2}=V_{T n}$ ), one can have

$$
I_{b}+I_{\text {in }}=K_{n} \cdot V_{G 2} \cdot V_{D S 2}
$$

A current-to-voltage converter is composed of M7 and M8 [7]. Assume that $\mathrm{M} 7$ and $\mathrm{M} 8$ are perfectly matched (i.e. $K_{p 7}=K_{p 8}=K_{p}$ and $V_{T p 7}=V_{T p 8}=V_{T p}$ ) and both are embodied in individual wells to avoid the body effect. If the supply voltages $V_{D D}=\left|V_{S S}\right|$, the gate voltage can be expressed as

$$
V_{G 2}=\frac{I_{8}-I_{7}}{2 K_{p}\left(V_{D D}-\left|V_{T p}\right|\right)}=\frac{I_{b}-I_{\text {in }}}{2 K_{p}\left(V_{D D}-\left|V_{T p}\right|\right)}
$$

Substituting (8) into (7) and comparing to (1) yields

$$
\begin{aligned}
V_{D S 2} & =\frac{2 K_{p}\left(V_{D D}-\left|V_{T p}\right|\right)}{K_{n}} \cdot \frac{I_{b}+I_{\mathrm{in}}}{I_{b}-I_{\mathrm{in}}} \\
& =\frac{2 K_{p}\left(V_{D D}-\left|V_{T p}\right|\right)}{K_{n}} \cdot \frac{1+I_{\mathrm{in}} / I_{b}}{1-I_{\mathrm{in}} / I_{b}} \\
& \simeq \frac{2 K_{p}\left(V_{D D}-\left|V_{T p}\right|\right)}{K_{n}} \cdot \exp \left(\frac{2}{I_{b}} I_{\text {in }}\right)
\end{aligned}
$$

Therefore, a pseudo-exponential function generator can be realised.

Experimental results: The proposed circuit has been fabricated in a $0.5 \mu \mathrm{m}$ CMOS process. The die photograph is shown in Fig. 3. The aspect ratios of all the transistors for the proposed circuit are $(W / L)=(1 \mu \mathrm{m} / 1 \mu \mathrm{m})$ and the experiments were performed with supply voltages $V_{D D}=\left|V_{S S}\right|=1.5 \mathrm{~V}$. The experimental results of the proposed pseudo-exponential function generator are shown in Fig. 4. With the reference current $I_{b}=40 \mu \mathrm{A}$, when $I_{\text {in }}$ varies from -30 to $15 \mu \mathrm{A}$, the output dynamic range could be more than $15 \mathrm{~dB}$ while the linearity error is within $\pm 0.5 \mathrm{~dB}$ and the power dissipation is less than $0.4 \mathrm{~mW}$. The experimental results confirm the theoretical analysis calculated by (9). 


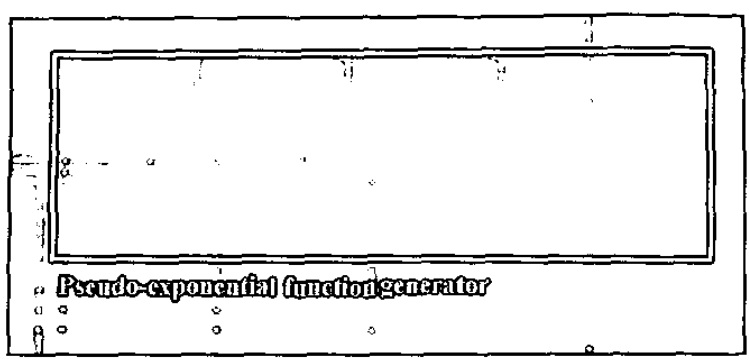

Fig. 3 Die photo of proposed exponential function generator

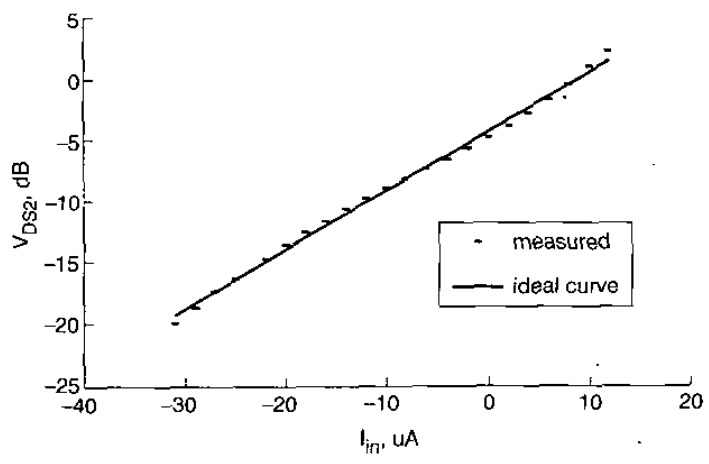

Fig. 4 Experimental results of proposed exponential function generator

Conclusion: A new CMOS pseudo-exponential function generator has been developed. Experimental results have been given to confirm the validity of the theoretical analysis. The proposed circuit is expected to be useful in the design of VGA and other analogue signal processing applications.

(C) IEE 2003

26 August 2002

Electronics Letters Online No: 20030013

DOI: 10.1049/el:20030013

Weihsing Liu and Shen-Iuan Liu (Department of Electrical Engineering and Graduate Institute of Electronics Engineering, National Taiwan University, Taipei, Taiwan 10617, Republic of China)

E-mail: 1si@cc.ee.ntu.edu.tw

\section{References}

1 MOTAMED. A., HWANG, C., and ISMAlL, M.: 'A low-voltage low-power wide range CMOS variable gain amplifier', IEEE Trans. Circuits Syst. II, Analog Digit. Signal Process., 1998, 45, (7), pp. 800-810

2 CHANG, C.C., and LIU, S.I.: 'Pseudo-exponential function using MOSFETs in saturation', IEEE Trans. Circtits Syst. Il, Analog Digit. Signal Process., 2000, 47, pp. 1318-1321

3 LIU, W., CHANG, C.C., and LIU, S.I.: 'Realisation of exponential V-I converter using composite NMOS transistors', Electron. Lett., 2000, 36, (1), pp. 8-10

4 VLASSIS, S.: 'CMOS current-mode pseudo-exponential function circuit', Electron. Lett., 2001, 37, (8), pp. 471-472

5 MOTAMED, A., and ISMAlL, M.: 'CMOS exponential current-to-voltage converter', Electron. Lett., 1997, 33, (12), pp. 998-1000

6 ABDELFATTAH, K.M., and SOLIMAN. A.M.: 'A new approach to realize variable gain amplificrs', J. Analog Integr. Circuits Signal Process., 2002, 30, pp. 257-263

7 BULT, K., and WALLINGA. H.: 'A class of analog CMOS circuits based on square law characteristics of an MOS transistor in saturation', IEEE J Solid-State Circuits, 1987, 22, pp. 357-365

\section{Combined radix $<2$ and $1.5 \mathrm{bit} / \mathrm{stage}$ pipelined analogue-to-digital converter}

\author{
B. Nejati and O. Shoaei
}

A new pipeline architecture that combines the radix $<2$ and tradition$1.5 \mathrm{bit}$ gain-stages is presented. The $10 \mathrm{bit}, 60 \mathrm{MHz}, 3 \mathrm{~V}$ pipeline analogue-to-digital converter has been designed in a $0.25 \mu \mathrm{m} ! \mathrm{P} 4 \mathrm{M}$ CMOS technology using digital self-calibration. The converte achieves more than $57 \mathrm{~dB}$ SNDR from a $3 \mathrm{~V}$ supply $(10 \%$ lowe than nominal $3.3 \mathrm{~V}$ ) within -40 to $+120^{\circ} \mathrm{C}$ temperature range.

Introduction: The $1.5 \mathrm{bit} / \mathrm{stage}$ pipelined analogue-to-digital converter (ADC) is almost the most power efficient architecture, but suffers from a complicated and long digital self-calibration period. This is a drawback because digital self-calibration has become more attractive for modern deep submicron CMOS technologies, in which the implementation of a digital circuit is becoming easier and faster. The radix $<2$ architecture has the advantage of having a simpler digital self-calibration but is more sensitive to circuit non-idealities. It also requires a higher number of stages compared to its $1.5 \mathrm{bit} / \mathrm{stage}$ counterpart, leading to larger power dissipation. In this Letter, a combined architecture of radix $<2$ and $1.5 \mathrm{bit} / \mathrm{stage}$ is presented. The few first stages make usc of radix $<2$ architecture and digital self-calibration, while the remaining stages use $1.5 \mathrm{bit} / \mathrm{stage}$ in which no calibration is employed. This architecture minimises the power dissipation, while requiring a simpler digital self-calibration. The presented 10 bit, $60 \mathrm{MHz}, 3.3 \mathrm{~V} \mathrm{ADC}$ consumes $50 \mathrm{~mA}$ in $3.0 \mathrm{~V}$ supply ( $10 \%$ lower than nominal voltage) and achieves $>57 \mathrm{~dB}$ SNDR in all process corner cases and temperature ranges. The IM3 of the converter is $>60 \mathrm{~dB}$.

Converter architecture: The first four stages have different radix factors as 1.4, 1.8, 1.75 and 1.75 while the remaining nine stages are traditional $1.5 \mathrm{bit} / \mathrm{stage}$. Overall there are 14 raw bits, four from the radix stages, and ten from the rest. The ADC output number associated with its full-scale level is 8180 out of which 3 bits are truncated resulting in 1024 levels (10 bit) at the output of the calibration/error-correction unit. Fig. 1 shows the corresponding weights of the raw bits, the ADC architecture, and the digitally calibrated stages. The ten least significant bits come from the overlapped 9 bit-pairs of the $1.5 \mathrm{bit} / \mathrm{stage}$ stages. Fig. 1 shows the full-scale range from $-V_{r e f}$ to $+V_{r e f}$ divided into 8180 codes by the ADC. The corresponding code of $-V_{r e f}$ is 0 and that of $+V_{r e f}$ is 8180. System level Monte-Carlo simulations using Matlab show that including non-idealities the converter achieves $<0.05 \mathrm{LSB}$ DNL and INL after calibration.

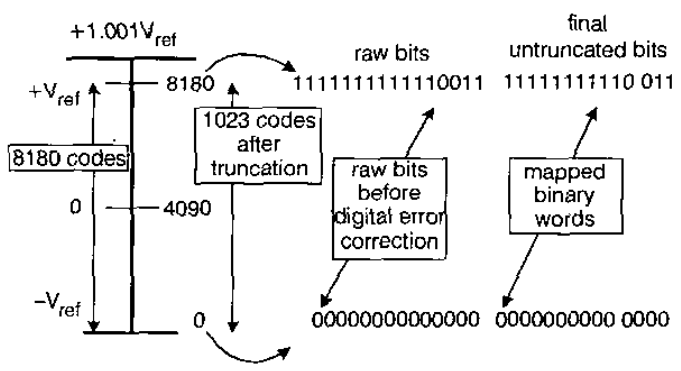

calibrated stage

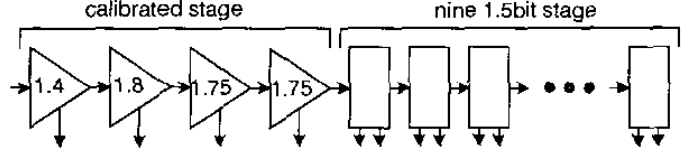

Fig. 1 Architecture of proposed ADC, bit weights, and full-scale distribution of codes

Radix $<2$ gain-stage architecture and calibration: Fig. 2 shows the input-output transfer function of the first four stages as well as the 